\title{
A reinforcement learning based collision avoidance mechanism to superposed LoRa signals in distributed massive loT systems
}

\author{
Takuma Onishi ${ }^{1, \text { a) }}$, Aohan $\mathrm{Li}^{1}$, Song-Ju Kim ${ }^{2}$, and Mikio Hasegawa ${ }^{1}$ \\ ${ }^{1}$ Department of Electrical Engineering, Tokyo University of Science \\ 6-3-1 Niijuku, Katsushika-Ku, Tokyo, 125-8585, Japan \\ ${ }^{2}$ Graduate School of Media and Governance, Keio University \\ 5322 Endo, Fujisawa, Kanagawa, 252-0882, Japan
}

a)t-onishi@haselab.ee.kagu.tus.ac.jp

Abstract: For Massive IoT systems, various Low Power Wide Area (LPWA) systems have been developed and deployed, i.e., LoRa, SigFox, etc. In this paper, to avoid destructive collisions when multiple IoT LoRa signals simultaneously received in the same channel, we propose a Successive Interference Cancellation (SIC) based collision avoidance mechanism by accessing channel using reinforcement learning for distributed massive IoT systems. Simulation results show the effectiveness of our proposed mechanism in terms of Frame Success Rate (FSR).

Keywords: massive IoT, collision avoidance, reinforcement learning, multiarmed bandit algorithm, successive interference cancellation

Classification: Wireless Communication Technologies

\section{References}

[1] M.A.B. Temim, G. Ferre, B. Laporte-Fauret, D. Dallet, B. Minger, and L. Fuche, "An enhanced receiver to decode superposed LoRa-like signals," IEEE Internet Things J., April 2020.

[2] T. Elshabrawy and J. Robert, "Analysis of BER and coverage performance of LoRa modulation under same spreading factor interference," Proc. 2018 IEEE 29th Annual International Symposium on Personal, Indoor and Mobile Radio Communications (PIMRC), pp. 16, Sept. 2018. DOI: 10.1109/pimrc. 2018.8581011

[3] H. Robbins, "Some aspects of the sequential design of experiments," Bulletin of the American Mathematical Society, vol. 58, pp. 527-535, 1952.

[4] J. Ma, S. Hasegawa, S.-J. Kim, and M. Hasegawa, "A reinforcement-learningbased distributed resource selection algorithm for massive IoT," Applied Science, vol. 9, 18, 2019.

[5] S.-J. Kim, M. Aono, and E. Nameda, "Efficient decision-making by volume conserving physical object," New Journal of Physics, vol. 17, 083023, 2015.

[6] G. Ferré and A. Giremus, "LoRa physical layer principle and performance analysis," Proc. 25th IEEE Int. Conf. Electron. Circuits Syst. (ICECS), pp. 65-68, Dec. 2018. DOI: 10.1109/icecs.2018.8617880 


\section{Introduction}

The number of Internet of Things (IoT) devices is predicted to grow to 40 billion by 2025. LoRa is one of the attractive solutions to Low Power Wide Area (LPWA) for massive IoT system. However, high traffic generated by the huge number of LoRa devices brings destructive collisions. To avoid destructive collisions when multiple IoT LoRa signals simultaneously received, Ref. [1] proposed a successive interference cancellation (SIC) algorithm to decode the superposed LoRa signals at receiver side. However, the proposed algorithm in [1] cannot effectively avoid collision when the number of IoT devices is large. The reason is that LoRa devices access channels randomly while superseded LoRa signals can be separated by SIC only when the power ratio between the strongest signal and the weakest signal is $1 \mathrm{~dB}$ or more [2]. When the power of the received LoRa signals in the same channel is similar, SIC may not work well in separating the received LoRa signals at receiver side. To solve this problem, we propose a SIC based collision avoidance mechanism by channel access using reinforcement learning for distributed massive IoT systems in this paper. In our proposed mechanism, LoRa devices select channel to transmit signals based on reinforcement learning method at transmitter side while SIC is used to separate superposed LoRa signals at receiver side. Channel selections by LoRa devices can be modeled as a Multi-Arm Bandit (MAB) problem [3]. The Tug-of-War (ToW) dynamics method that can effectively solve MAB problems with low computation complexity $[4,5]$ is used to solve the modeled channel selection MAB problem. We verify the performance of the proposed method by comparing frame success rates with the conventional methods.

\section{System model}

We consider uplink communications between LoRa devices and gateways (GWs) in distributed Massive IoT system. The chirp signal transmitted by the $i$-th LoRa device at time $p T$ can be expressed as follows,

$$
f_{i}(t-p T)=\frac{1}{2 \pi} \frac{d \varphi_{i}(t-p T)}{d t}
$$

where $T$ is the symbol time period. $\varphi_{i}(t-p T)$ is

$$
\varphi_{i}(t-p T)= \begin{cases}2 \pi\left[\frac{B}{2 T} t^{2}+\frac{m_{i}(p)}{T} t\right] & t \in\left[p T-\frac{T}{2}, p T+\frac{T}{2}-\gamma_{i}(p)\right) \\ 2 \pi\left[\frac{B}{2 T} t^{2}+\left(\frac{m_{i}(p)}{T}-B\right) t\right] & t \in\left[p T+\frac{T}{2}-\gamma_{i}(p), p T+\frac{2}{T}\right) . \\ \gamma_{i}(p)=\frac{m_{i}(p)}{B}\end{cases}
$$

where $m_{i}(p) \in[0, \ldots, M-1]$ is the symbol transmitted at time $p T, M=2^{S F}, S F$ is spreading factor, which means the number of bits per symbol, and $B=\frac{2^{S F}}{T} . s_{i}(t)$ is the complex envelope of the signal transmitted by the $i$-th LoRa device, which can be expressed as follows,

$$
s_{i}(t)=\sum_{p \in \mathbb{Z}} e^{j \varphi_{i}(t-p T)}
$$

The de-chirping sequence, sampled at $T_{S}=\frac{1}{B}$, can be expressed as follows. 


$$
d(n)=\sum_{p \in \mathbb{Z}} e^{-j \varphi\left(n T_{s}-p T\right)}
$$

The received signal can be written as follows.

$$
y(n)=\sum_{i=1}^{U} \sqrt{P_{i}} s_{i}(n) e^{-j 2 \pi n T_{s}}+w(n)
$$

where $U$ is the number of LoRa devices, $\sqrt{P_{i}}$ is the received signal power of the $i$-th LoRa device, and $w(n)$ is white Gaussian noise.

The signal corresponding to the $p$-th symbol processing obtained after the fast Fourier transform based on the LoRa symbol detection principle is shown as follows.

$$
\begin{gathered}
Y(k, p)=\frac{1}{\sqrt{M}} \sum_{n=0}^{M-1} z(n, p) e^{-j 2 \pi \frac{n k}{M}} \\
z(n, p)=y(n, p) d(n, p)
\end{gathered}
$$

where $y(n, p)=y(n)$ and $d(n, p)=d(n) \forall n \in[p M,(p+1) M-1], k \in[0, \ldots, M-1]$. Equation (7) can be transformed as follows based on the Ref. [6].

$$
z(n, p)=\sum_{i=1}^{U_{p}}\left(\sqrt{P_{i}} e^{j 2 \pi n \frac{m_{i}\left(p_{i}-1\right)}{M}}+\sqrt{P_{i}} e^{j 2 \pi n \frac{m_{i}\left(p_{i}\right)}{M}}+w(n)\right)
$$

where $U_{p} \in[1, \ldots, U]$ is the number of $p^{t h}$ received signals. The received signal $z_{s}\left(n, p_{s}\right)$ synchronized \& de-chirped of the strongest signal is shown as follows.

$$
z_{s}\left(n, p_{s}\right)=\sqrt{P_{S}} e^{j 2 \pi n \frac{m_{s}\left(p_{s}\right)}{M}}+\sum_{i=1}^{U_{p_{s}-1}} z_{i}\left(n, p_{s}\right)+w(n)
$$

The estimation of the transmitted $p_{s}$-th symbol by the $s^{t h}$ SIC iteration is

$$
\hat{m}_{s}\left(p_{s}\right)=\arg \max _{k}\left(\left|Y^{s}\left(k, p_{s}\right)\right|\right)
$$

The signal reconstruction can be written as follows.

$$
\begin{gathered}
z_{s+1}\left(n, p_{s}\right)=z_{s}\left(n, p_{s}\right)-\hat{z}_{s}\left(n, p_{s}\right) \\
\hat{z}_{s}\left(n, p_{s}\right)=\sqrt{P_{s}} e^{j 2 \pi n \frac{\hat{m}_{s}\left(p_{s}\right)}{M}}
\end{gathered}
$$

The transmitted signal can be reconstructed at receiver side using SIC iteratively based on Eq. (10) and Eq. (11), which can be summarized as follows. Firstly, in the $s^{t h}$ SIC iteration, the $p^{t h}$ chip signal of the transmitted LoRa symbols $\hat{m}_{s}\left(p_{s}\right)$ can be estimated by Eq. (10). Then, the $p^{t h}$ chip signal can be reconstructed based on the estimated $\hat{m}_{S}\left(p_{S}\right)$ and the received power of the $p^{t h}$ chip signal $P_{S}$ using Eq. (13). All of the transmitted LoRa symbols can be reconstructed by repeat the SIC iteratively summarized as above. 


\section{Proposed collision avoidance mechanism}

In our proposed collision avoidance mechanism, ToW is used to channel selection by LoRa devices at the transmitter side while SIC is used by GW at receiver side.

Channel selection in IoT systems was modeled as the MAB problem in [4]. The MAB problem is a problem that maximizes the total reward obtained by playing multiple slot machines with unknown reward probabilities in a limited number of trials [3]. In the proposed method of Ref. [4], channels are modelled as slot machines in the MAB problem while the reward is obtained based on whether ACK frame is received at transmitter side. The ToW dynamics method is used to solve the modelled MAB problem, with low computation complexity [5]. In ToW dynamic, $Q$ value is updated as follows.

$$
\begin{aligned}
& Q_{k}(t)=\alpha Q_{k}(t-1)+\Delta Q_{k}(t) \\
& \Delta Q_{k}(t)=\left\{\begin{array}{l}
+1, \quad \text { ACK is received } \\
-\omega, \quad \text { ACK is not received }
\end{array}\right.
\end{aligned}
$$

where $\alpha(0 \leq \alpha \leq 1)$ is defined as forgetting coefficient. The optimum $\omega$ is derived in [5], which can be written as:

$$
\omega=\frac{P_{1 s t}(t)+P_{2 n d}(t)}{2-\left(P_{1 s t}(t)+P_{2 n d}(t)\right)}
$$

where $P_{1 s t}$ and $P_{2 n d}$ represent the top two reward probabilities at time $t$. Displacement $X_{k}$ based on Equations (14) and (15) is used for decision making (channel selection in the proposed method in [4]), which can be expressed as follows.

$$
\begin{gathered}
X_{k}(t)=Q_{k}(t-1)-\frac{1}{K-1} \sum_{k^{\prime} \neq k}^{K} Q_{k}(t)+\operatorname{osc}_{k}(t) \\
\operatorname{osc}_{k}(t)=A \cos \left(\frac{2 \pi t}{n}+\frac{2(k-1) \pi}{n}\right)
\end{gathered}
$$

Here $\operatorname{osc}_{k}(t)$ represents vibration. Note that $A$ is an amplitude parameter, which is fixed at $A=0.5$ in this paper. The slot machine to be played at time $t+1$ is determined to be machine $k$, i.e., $k=\arg \max X_{k}(t+1)$.

\section{Performance evaluation}

In this Section, we evaluation our proposed collision avoidance mechanism in terms of FSR, which is defined as follows,

$$
\mathrm{p}_{\mathrm{k}}(\mathrm{t})=\frac{\text { Number of successful transmissions using channel } k \text { by time } t}{\text { Number of transmissions using the channel } k \text { by time } t} .
$$

To verify the effectiveness of our proposed mechanism, we compare it to other two settings, which are "SIC only" and "ToW only". In the "SIC only", each IoT device uses the channel that is allocated to its nearest GW.

Figures 1 and 2 show simulation results in case that there are 300 and 1200 LoRa devices, randomly placed in a circle with a radius of $800 \mathrm{~m}$, respectively. $3 \mathrm{GWs}$ are placed at the center of the circle. 1 channel is assigned to each GW. The spread factor of the LoRa devices is set as 8 . The simulation period is set as $100 \mathrm{~s}$. To 


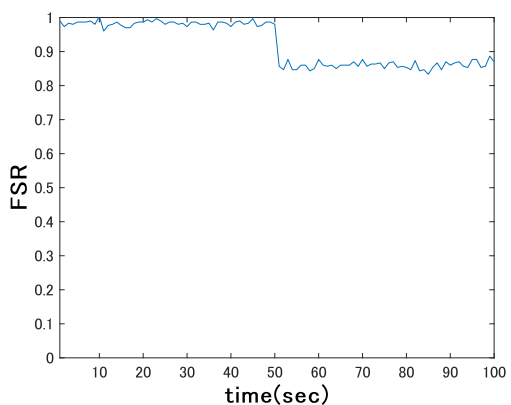

(a) SIC

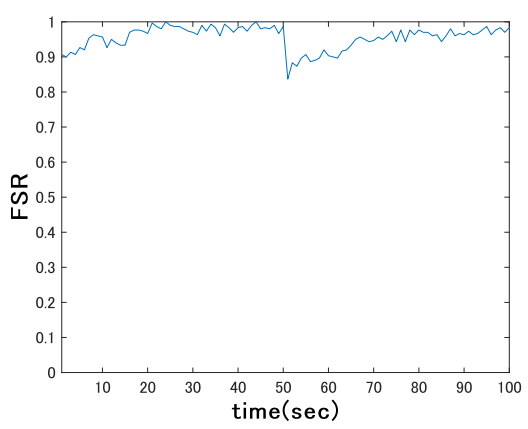

(c) $\mathrm{SIC}+\mathrm{ToW}$

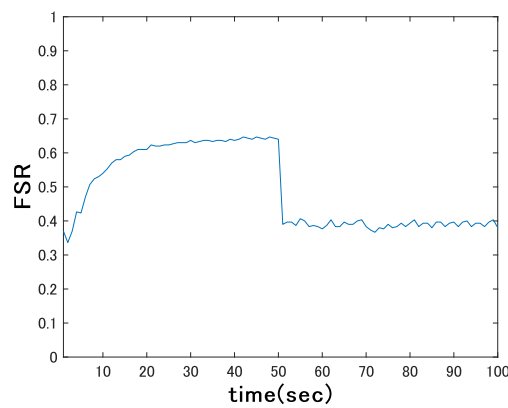

(b) ToW

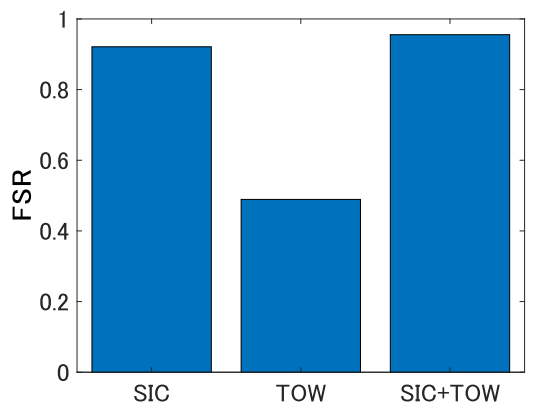

(d) Comparison of average FSR

Fig. 1. 300 LoRa devices performance evaluation

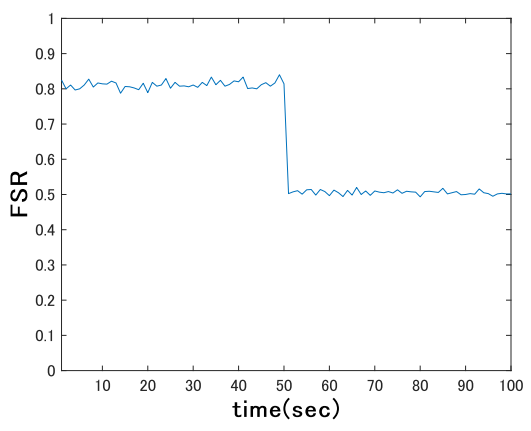

(a) SIC

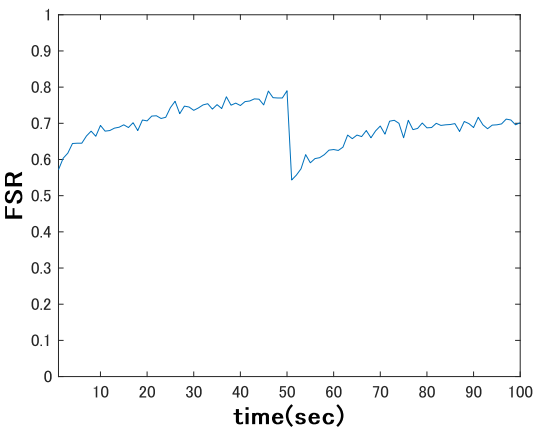

(c) $\mathrm{SIC}+\mathrm{ToW}$

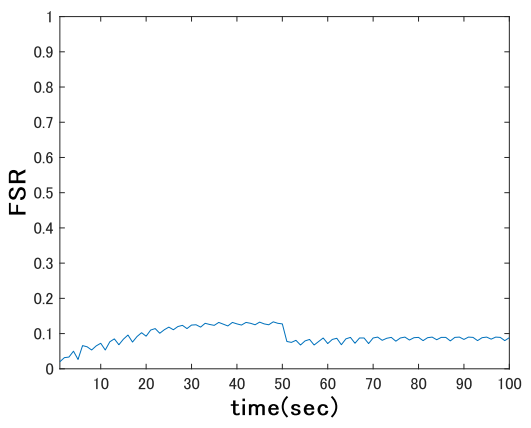

(b) ToW

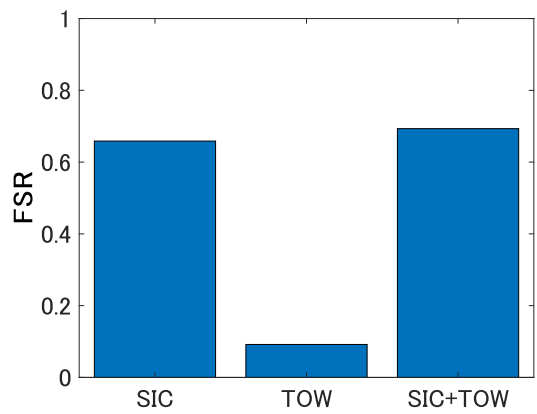

(d) Comparison of average FSR

Fig. 2. 1200 LoRa devices performance evaluation 
investigate the performance in dynamic situation, we add the traffic load to GW3 during $51 \mathrm{~s}$ to $100 \mathrm{~s}$.

From Figs. 1(a) and 2(a), we can see that FSR is reduced during $51 \mathrm{~s}$ to $100 \mathrm{~s}$ for the "SIC only". The reason is that the number of LoRa devices with same received power that access the same channel becomes larger, which leads to the failure of decode by SIC. From Figs. 1(b) and 2 (b), we can see that received superposed LoRa signal may not be decoded successfully without SIC in "ToW only". From Figs. 1(c) and 2(c), we can see that our proposed collision avoidance mechanism not only can select appropriate channel, but also can decode the superposed LoRa signal successfully. Figs. 1(d) and 2(d) shows the comparison of these settings in average FSR, from which we can see the effectiveness of our proposed collision avoidance mechanism.

\section{Conclusion}

In this paper, we propose a reinforcement learning based collision avoidance mechanism to massive IoT systems. Simulation results show the effectiveness of our proposed mechanism in terms of FSR. Furthermore, reinforcement learning showed that it can be adapted to a dynamic wireless environment. 